\title{
Problems Solving of Cell Subscribers based on Expert Systems Neural Networks
}

\author{
Ahmad AbdulQadir AlRababah \\ Faculty of Computing and Information Technology, King Abdulaziz University, \\ Rabigh 21911, Kingdom of Saudi Arabia
}

\begin{abstract}
With the growing demand for telecommunications services, the number of calls to telecommunications companies related to issues of using services, setting up and maintaining equipment, as well as resolving possible problems arising in the process of using services is also growing. From the point of view of system analysis, the problem is the mismatch between the existing and the required (target) state of the system for a given state of the environment at the moment in time. Based on this definition, we consider the problem of the subscriber of the cellular network a mismatch between the existing and the required state of the cellular network in this state of the environment at the moment in time. The state of the cellular network is characterized by the functioning of all devices, the proposed range of services. A short time for analyzing problem situations and making decisions, a large amount of information characterizing the current situation, the difficulty of solving poorly formalized and poorly structured tasks in the absence of complete and reliable information about the state of the cellular communication network and the functioning of its elements lead to a mismatch of human capabilities to effectively solve these problems. In this regard, the development and implementation of a precedent based neural network expert system for solving the problems of subscribers of a cellular communication network is an urgent scientific and technical task.
\end{abstract}

Keywords-Neural network expert system; telecommunications companies; system analysis; cellular network; structured tasks; reliable information; human capabilities; telecommunications services

\section{INTRODUCTION}

Development of precedent based neural network expert system on an integrated approach to the problem of effective relationship management with subscribers of a cellular communication network [4,17,31], including the use of expert systems technologies, neural networks, case-based reasoning, as well as the creation of models [10,24,37], algorithms and support programs for decision-makers interacting with subscribers of a cellular communication network.

Research Objectives of this manuscript are considered analysis of the most popular systems for managing cellular networks $[5,19,32,40]$ and customer relationships; research of existing methods and techniques for decision support for tasks of managing relationships with subscribers of cellular communication networks; analysis of various technologies of intelligent systems, methods of their interaction and combination [15,29]; building mathematical models of precedent based neural network expert system components: a production fuzzy knowledge base about subscriber problems[16,30,42], a fuzzy controller based on a neural network, a knowledge base of precedents for problems; development of a complex of algorithms: processing subscriber applications, finding solutions to subscribers' problems based on precedents and using a fuzzy neural network[3,18,34]; creation of a neural network expert system based on precedents for solving problems of subscribers of a cellular communication network; study of the effectiveness of the developed precedent based neural network expert system using the following groups of indicators: functional suitability, efficiency, reliability, cost-effectiveness of the system[2,8,39]; development and implementation in trial operation of the precedent based neural network expert system software[6,13] to solve the problems of cellular network subscribers.

Scientific novelties of this research are mentioned in the existing approaches to solving the problems of managing the relationships of operator companies with subscribers of cellular networks[11,25]; theoretically substantiated a new approach to building an intelligent system to solve the problems of subscribers of a cellular communication network, based on the integrated use of technology expert systems, neural networks, fuzzy logic and reasoning based on precedents; software was developed for building a precedent based neural network expert system, including: a production fuzzy knowledge base about problems of subscribers, a fuzzy controller based on a neural network[7,20], a knowledge base of precedents for problems; algorithms have been created for finding solutions to subscribers' problems based on precedents and using a fuzzy neural network; a neural network expert system based on precedents was developed to solve the problems of subscribers of a cellular communication network[21,33,38]; experimental studies were conducted to verify the effectiveness of the developed system.

The practical value of this research was explored as a first version of the software neural network expert system based on precedents for solving the problems of subscribers of a cellular network after testing and evaluating specialists in 2009, where it was used to apply as an integrated approach to the development of precedent based neural network expert system, methods for building knowledge bases of fuzzy products and use cases[1,14,41], algorithms for finding solutions based on use cases and a neural networks.

\section{RESEARCH FRAMEWORK}

The first stage of the research will focus on analyzes methods and software tools for solving problems associated with servicing subscribers of cellular communication 
networks[9,22]. The study showed that making decisions in the field of telecommunication network management, and in particular, in solving problems of servicing subscribers of such networks is a complex and multi-criteria task [23,28]. One of the bottlenecks in its solution is the procedure for finding the cause of problems with the provision of cellular services to the subscriber. This task requires a minimum of time to solve it and a maximum of reliability of the found solution to the identified problem $[12,35]$. The complexity of the problem also lies in the fact $[26,38,43]$ that the state and dynamics of the processes of functioning of a cellular communication network cannot be unambiguously described using clear mathematical models.

Based on the analysis of operators serving cellular network subscribers, it is concluded that it is necessary to use intelligent systems (IS) [7,39] that combine previously accumulated experience in the field of operation of a cellular communication network. As a result of research on various IS technologies and methods of their interaction, the feasibility of hybridization of various intellectual components is substantiated $[5,27,36]$. This stage of the work concludes with the goals and objectives of the study.

\section{Proposed Methodology}

The next stage of this research discusses the theoretical foundations and resolves issues of developing mathematical support for a precedent based neural network expert system to solve the problems of subscribers of a cellular communication network. The mathematical description of a neural network expert system based on precedents has the form:

$$
N E S^{p}=<K B, K B_{p}, A(p), R^{\prime \prime}, I^{n p}, I^{n 2}, I^{p}>
$$

In a precedent based neural network expert system, the knowledge base contains knowledge in the form of products $K B$ and in the form of precedents $K B_{p}, R^{\prime \prime}$ - systemic relations of IS. The search for solutions is divided into a neural network $I^{n 2}$ and case law $I^{p}$ with $A(p)$ - an algorithm for determining similar use cases. Neural network training $I^{n p}$ is based on data from precedents.

A model of a system for solving subscriber servicing problems is presented as a nonlinear object (Fig. 1) with many inputs $\left\{x_{i}\right\}$ and output variables $\left\{y_{k}\right\}$ :

$$
\left\{\begin{array}{l}
\left\{x_{i}\right\}, i=\overline{1, n} ; \\
\left\{y_{k}\right\}=f_{y}\left(x_{1}, x_{2}, \ldots, x_{n}\right), k=\overline{1, q}
\end{array}\right.
$$

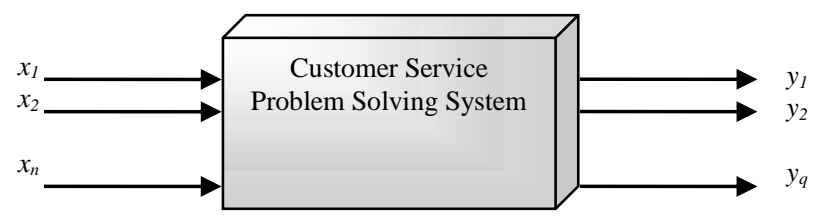

Fig. 1. Model of a System for Solving Problems of Customer Service.
Input variables are characteristics of the problem that the subscriber has. The output variables are the causes of the problem. Input $\left\{x_{i}\right\}, i=\overline{1, n}$ and outputs $\left\{y_{k}\right\}, k=\overline{1, q}$ variables can take only qualitative values, and the set of all possible values of these variables is known

$U=\left\{u_{j}, u_{j+1}, \ldots, u_{m}\right\}$

Where $u_{j}$ - score corresponding to the lowest value of the input $x_{i}$ or outputs $y_{k}$ variable; $u_{m}$ - score corresponding to the largest input value $x_{i}$ (or output value $y_{k}$ ) variable $m$ is the power of the set $U$.

We accept that the vector $X^{*}=\left\{x_{1}^{*}, x_{2}^{*}, \ldots, x_{n}^{*}\right\}$ fixed values of input variables of the considered system model, where $x_{i}^{*} \in U, i=\overline{1, n}$.

The task of finding a solution is based on the vector $X^{*}$ define output vector $Y^{*}=\left\{y_{1}^{*}, y_{2}^{*}, \ldots, y_{q}^{*}\right\}$. Input and output variables will be considered as linguistic variables defined on universal sets $U$.

To evaluate linguistic variables, qualitative terms from the following term set are used:

$$
A=\left\{a_{j}, a_{j+1}, \ldots, a_{m}\right\} \text {, }
$$

Where $A$ is the term set of variables $x_{i}$ and $y_{k}, a_{j}-\mathrm{j}$-th linguistic term of a variable $x_{i} y_{k}, i=\overline{1, n}, j=\overline{1, m}$, $k=\overline{1, q}$.

Linguistic terms $a_{j}, a_{j+1}, \ldots, a_{m}$ are calculated as follows: $a_{j}=\sum_{p=1}^{l} \mu^{a_{j}}\left(u_{j}^{p}\right) / u_{j}^{p}$, where $\mu^{a_{j}}\left(u_{j}^{p}\right)$ - degree of membership of an element $u_{j} \in U$ of term $a_{j} \in A$, $p=\overline{1, l}, j=\overline{1, m}$. The task of constructing membership functions of elements $\boldsymbol{u}_{j} \in \boldsymbol{U}$ term set $A=\left\{a_{j}, a_{j+1}, \ldots, a_{m}\right\}$ comes down to determining degrees of belonging $\mu^{a_{j}}\left(u_{j}^{p}\right)$ for all $p=\overline{1, l}$, $j=\overline{1, m}$.

In accordance with (1), the MIMO structure (Multiple Input - Multiple Output) of a fuzzy knowledge base of the form:

If $\left(x_{1}=a_{1}{ }^{\prime}\right)$ and $\left(x_{2}=a_{2}{ }^{\prime}\right)$ and $\ldots$ and $\left(x_{n}=a_{m}{ }^{\prime}\right)$, then $\left(y_{1}=b_{1}{ }^{\prime}\right)$ and $\left(y_{2}=b_{2}{ }^{\prime}\right)$ and $\ldots$ and $\left(y_{n}=b_{m}{ }^{\prime}\right)$

Where $l$ is the rule number, $l=\overline{1, L}$, $\mathrm{L}$ is the number of rules, $a_{j}^{l}$ and $b_{j}^{l}$ - fuzzy terms to evaluate the input variable 
$x_{i}$ and output variable $y_{k}, \quad(i=\overline{1, n}, \quad j=\overline{1, m}$, $k=\overline{1, q}$ ) in the $l$-th rule, respectively.

We transform the system of logical statements (4) using operations $\cup$ (or), $\cap$ (and):

$\bigcup_{p=1}^{t_{k}}\left[\bigcap_{i=1}^{n}\left(x_{i}=a_{j}^{k p}\right)\right] \rightarrow \bigcup_{p=1}^{t_{k}}\left[\bigcap_{k=1}^{q}\left(y_{k}=b_{j}^{k p}\right)\right]$

Where

$$
j=\overline{1, q}, k=\overline{1, q}, p=\overline{1, t_{k}}
$$

\section{IMPLEMENTATIONS AND EXPEREMENTAL RESULTS DISCUSSIONS}

To implement the process of extracting knowledge from a fuzzy knowledge base, a neuro-fuzzy logical inference mechanism is used in the form of a fuzzy controller based on a neural network - NNFLC (Neurons Network Fuzzy Logic Controller) (Fig. 2).

Structurally, NNFLC is a multilayer network for direct signal propagation, and different layers perform different functions.

Layer 1: represents membership functions implemented as radial basis neurons: $y_{i}^{(1)}(x)=\exp \left[-\left(x_{i}-c_{i}\right)^{2} / 2 \cdot \sigma_{i}^{2}\right]$.

Layer 2: models the and- conditions of the rules: $y_{i}^{(2)}=\min \left[y_{1}^{(1)}, \ldots, y_{n}^{(1)}\right]$.

Layer 3: is an OR combination of rules with identical terms in consequents: $y_{i}^{(3)}=\max \left[y_{1}^{(2)}, \ldots, y_{n}^{(2)}\right]$. In the training mode, the layer adjusts the parameters of the membership functions of the output variables. In operating mode, forms an output.

Layer 4: in operating mode, neurons perform defuzzification: $z_{i}^{(4)}=\sum_{j} \omega_{j i} \cdot y_{i}^{(3)}$. In training mode, this is an additional input that performs normalization, which allows you to configure the membership function of the output variable: $y_{i}^{(4)}\left(z_{i}^{(4)}\right)=\sum_{j} y_{i}^{(3)} \cdot\left[\omega_{j i} / \sum_{j} \omega_{j i}\right]$.

The structure of a fuzzy neural network NNFLC is initialized on the basis of the formation of a complete matrix of rules.

The mathematical definition of the knowledge base of precedents has the form: $\boldsymbol{B P}=\left\{\left\langle\pi_{1}, \pi_{2}, \ldots, \pi_{n}\right\rangle, K, I\right\}$, where $\left\langle\pi_{1}, \pi_{2}, \ldots, \pi_{n}\right\rangle$ - a lot of precedents, $I$ - a set of index terms that determine whether the precedent belongs to class $K$.
Next stage of this research discusses the architectural features of precedent based neural network expert system (Fig. 3 ) to solve the problems of subscribers of a cellular communication network. Fig. 3 shows the structural diagram of the system. The input data for the precedent based neural network expert system is information from the application submitted by the network subscriber. The application contains general data, technical parameters, a list of actions carried out on the application.

layer1 layer2 layer3 layer 4

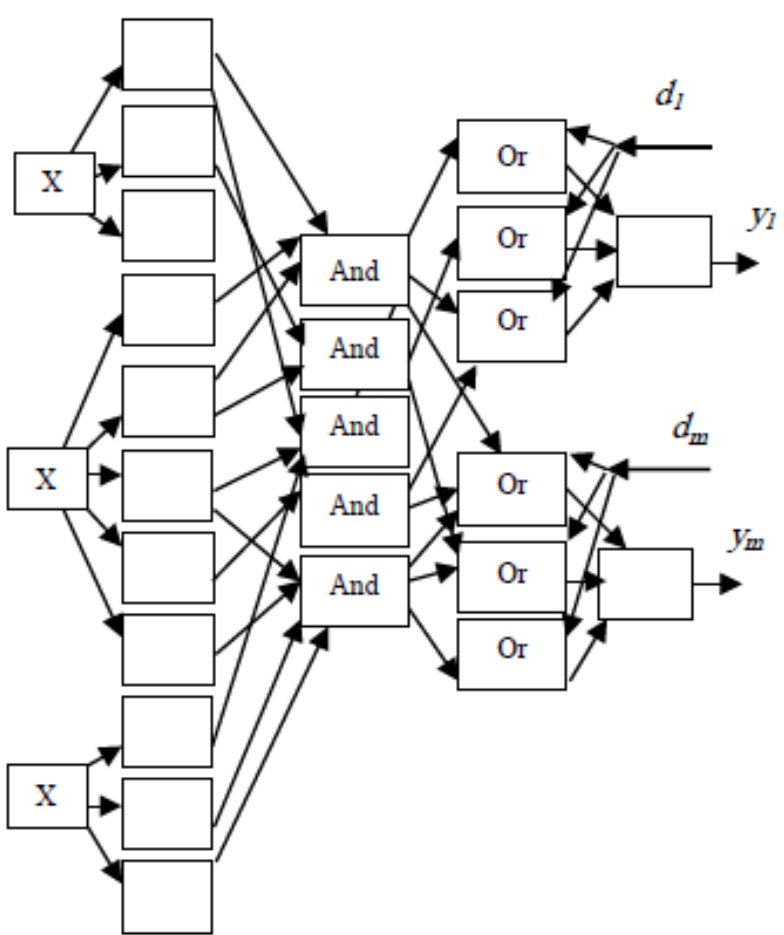

Fig. 2. The Structure of the Neuro-Fuzzy Controller NNFLC.

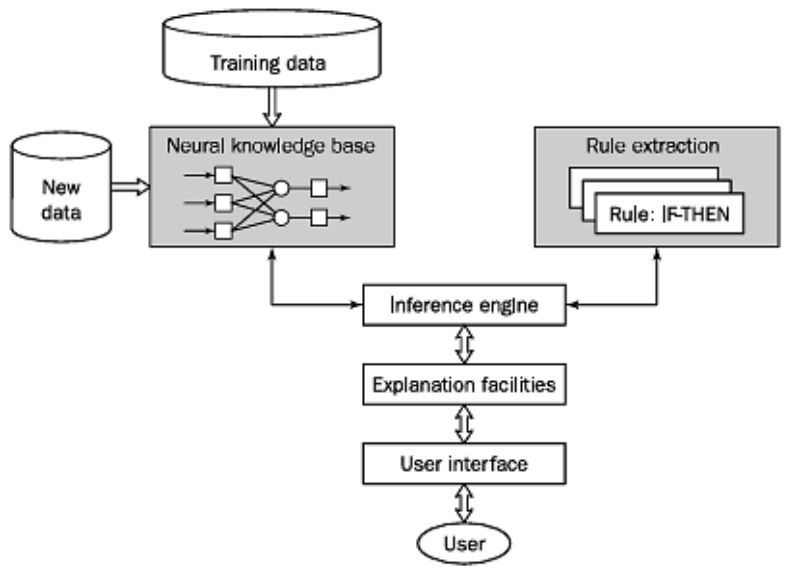

Fig. 3. Structure of Neural Network Expert System. 
An algorithm for extracting precedents from the knowledge base using the Euclidean metric is developed. The input to the algorithm is:

1) a description of the subscriber's problem $P=\left\{p_{1}, p_{2}, \ldots, p_{n}\right\}$, including $n$ values of parameters characterizing the situation;

2) $B P$ is a non-empty set of precedents;

3) $W=\left\{W_{1}, W_{2}, \ldots, W_{n}\right\}$ - weight (importance factors) parameters;

4) $M$ - the number of precedents under consideration from the knowledge base,

5) $K$ - threshold value of the degree of similarity.

Output: a lot of precedents $S P$, which have a degree of similarity greater than (or equal to) the threshold value $K$.

Step 1. $S P=\varnothing, j=1$ and go to the next step.

Step 2. If $j \leqslant M$ we choose the use case $A_{j}$ from the set $B P$ $\left(A_{j} \in B P\right)$ and go to step 3 , otherwise the use cases are considered and go to step 7.

Step 3. We calculate the distance according to the Euclidean metric between the selected use case $A j$ and the current situation $P$, taking into account the importance factors of the parameters: $D\left(P, A_{j}\right)=\sqrt{\sum_{i=1}^{n}\left(a_{i}-p_{i}\right)^{2}} \cdot w_{i}$

Step 4. We calculate the distance according to the Euclidean metric for the boundary values of the parameters: $D_{\max }(P)=\sqrt{\sum_{i=1}^{n}\left(p_{\max }-p_{\min }\right)^{2}} \cdot w_{i}$

Step 5. In this step, we calculate the degree of similarity $S(A, P)=\left(1-D / D_{\max }\right) \quad$ or as a percentage $S(A, P)=\left(1-D / D_{\max }\right) \cdot 100 \%$, if the threshold value of $\mathrm{K}$ is set in percent, and go to step 6 .

Step 6. If $S(A, P) \geq K$, then this precedent $A j$ is added to the result set $S P\left(A_{j} \in S P\right)$, means we extract this precedent from the knowledge base. After checking, $j=j+1$ and go to step 2 .

Step 7. If $S P=\varnothing$, then no precedents for the current problem situation were found and go to step 9 with a message for the operator about the need to reduce the threshold value $K$, otherwise the precedents for the current situation were successfully extracted and go to the next step.

Step 8. The found precedents are sorted in decreasing order of similarity with the current situation and presented to the operator.

Step 9.The end (completion of the algorithm).

Neural network training is as follows:

In step 1. A training sample is set, consisting of many examples of the following form: $\left(x_{1}^{(k)}, x_{2}^{(k)}, x_{3}^{(k)}, \ldots, x_{n}^{(k)}, y_{1}^{(k)}, y_{2}^{(k)}, y_{3}^{(k)}, \ldots, y_{q}^{(k)}\right)$ $k=\overline{1, K}$, where $x_{i}^{(k)}$ - values of input variables $x_{i}$ $i=\overline{1, n}$ ) and $y_{j}^{(k)}$ - values of output variables $y_{j}$ $j=\overline{1, q}$ )in k-th example; $K$ is the total number of examples in the training set. The values of the input and output variables are determined by the membership functions of the corresponding terms: $\left(\mu_{a_{1}}\left(x_{1}^{(k)}\right), \ldots, \mu_{a_{n}}\left(x_{n}^{(k)}\right), \mu_{b_{1}}\left(y_{1}^{(k)}\right), \ldots, \mu_{b_{q}}\left(y_{q}^{(k)}\right)\right)$, $k=\overline{1, K}$.

We introduce the notation $x_{i}^{(k)}$ and $y_{j}^{(k)}$ as values of membership functions of the terms of input and output variables, respectively.

In step 2. Configuring the parameters of membership functions includes determining the centers $c_{i}$ and widths $\sigma_{i}$ for membership functions represented by shape functions: $y_{i}^{(1)}(x)=\exp \left[\frac{-\left(x_{i}-c_{i}\right)^{2}}{2 \cdot \sigma_{i}^{2}}\right]$

The training data self-organization algorithm is used, which automatically divides the space into clusters. The center $c_{i}$ of the cluster is identified with the center of the radial basis function. The preliminary selection of centers is carried out randomly on the basis of uniform distribution.

The matrix of bond weights $\omega_{j i}$ is specified following the conditions: $\left\{\begin{array}{c}\omega_{i i}=1, \\ -\frac{1}{T-1}<\omega_{j i}<0, i \neq j,\end{array}\right.$ where $\mathrm{T}$ is the number of neurons in the input layer.

After presentation of the $\mathrm{k}$-th vector $x^{(k)}$ the center is selected from the training set $c_{i}$ closest to $x^{(k)}$ according to the Euclidean metric: $\left\|x^{(k)}-c_{i}\right\|=\sqrt{\sum_{t=1}^{T}\left(x_{t}-c_{i}\right)^{2}}$.

This center is subject to refinement in accordance with the winner algorithm (WTA algorithm):

$$
c_{i}(k+1)=c_{i}(k)+\eta(t) \cdot\left[x^{(k)}-c_{i}(k)\right], \text { where }
$$
$\eta(t)$ - monotonously decreasing level of training. Other centers do not change. All training vectors $x^{(k)}$ presented several times in random order until the stabilization of the values of the centers.

Width Adjustment $\sigma_{i}$ carried out heuristically, on the principle of "first closest neighbor": $\sigma_{i}=\left(\sigma_{i}-\sigma_{\omega}\right) / \lambda$, where $\lambda$ is the overlap parameter. The outputs of each layer are calculated by the formulas:

$$
\begin{aligned}
& y_{i}^{(2)}=\min \left[y_{1}^{(1)}, \ldots, y_{n}^{(1)}\right], \\
& y_{i}^{(3)}=\max \left[y_{1}^{(2)}, \ldots, y_{n}^{(2)}\right], \\
& y_{i}^{(4)}=\sum_{j} y_{i}^{(3)} \cdot\left[\omega_{j i} / \sum_{j} \omega_{j i}\right]
\end{aligned}
$$


Winner Algorithm Looks For Weight Matrix $\omega_{j i}$, which evaluates the quality of relations between the left and right parts of the rules: $\omega_{j i}(n+1)=\omega_{j i}(n)-\eta \cdot \Delta \omega$, where $\Delta \omega=y_{j}^{(3)} \cdot\left(y_{i}^{(2)}-\omega_{j i}\right)$.

In step 3. The combination of rules is carried out with the participation of an expert.

In step 4. Final configuration of membership functions is performed using the error back propagation algorithm for the error function $e_{i}=\left(y_{i}^{(4)}-d_{k}\right)^{2}$ regarding vectors $x^{(k)}$.

For $\mathrm{K}$ learning pairs, the objective error function is defined as:

$$
E=\frac{1}{2} \sum_{k=1}^{K}\left(y_{k}^{(4)}-d_{k}\right)^{2}=\frac{1}{2} \sum_{k=1}^{K}\left[\sum_{t=0}^{T} \omega_{i} \cdot y_{t}^{(4)}\left(x_{k}\right)-d\right.
$$

The magnitude of the error determines the gradient vector of the objective function relative to specific centers $c_{i j}$ and width

$$
\begin{aligned}
& \sigma_{i j}: \\
& c_{i j}(n+1)=c_{i j}(n)-\eta \cdot \frac{\partial E}{\partial c_{i j}(n)}, \\
& \sigma_{i j}(n+1)=\sigma_{i j}(n)-\eta \cdot \frac{\partial E}{\partial \sigma_{i j}(n)} .
\end{aligned}
$$

Repeated training cycles lead to complete and fast network learning.

The search for a solution to the subscriber's problem using the neural network mechanism is carried out according to the created algorithm (Fig. 4).

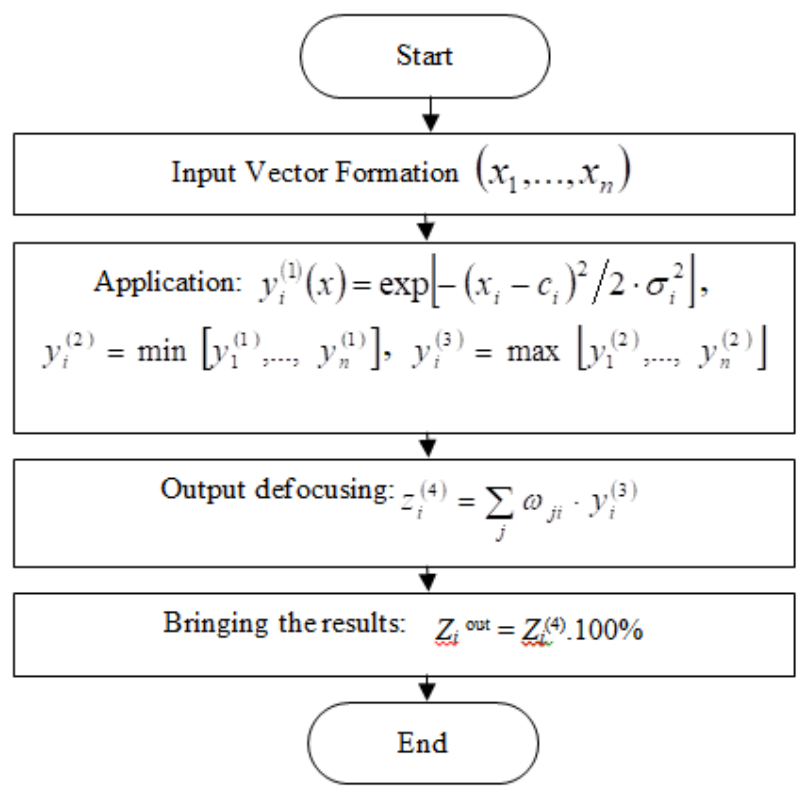

Fig. 4. Algorithm for Neural Network Search for a Solution to a Problem.
The next main stage of this research discusses the features of the software implementation of a precedent based neural network expert system. The choice of the object-oriented programming language Java as a part of the Microsoft Visual Studio with a program development environment as the main precedent based neural network expert system programming tool is substantiated. The modern database development tools are analyzed and the choice is made in favor of the Microsoft SQL Server DBMS.

A production fuzzy knowledge base has been developed for a system for solving problems of cellular network subscribers, training data sets have been generated for a neural network search mechanism. A software implementation of a fuzzy neural network was made, an algorithm for training and finding a solution using a neural network was implemented. A database of customer applications has been developed. A precedent presentation form is defined, a precedent knowledge base is built (Fig. 5), and a software implementation of the use case search is implemented.

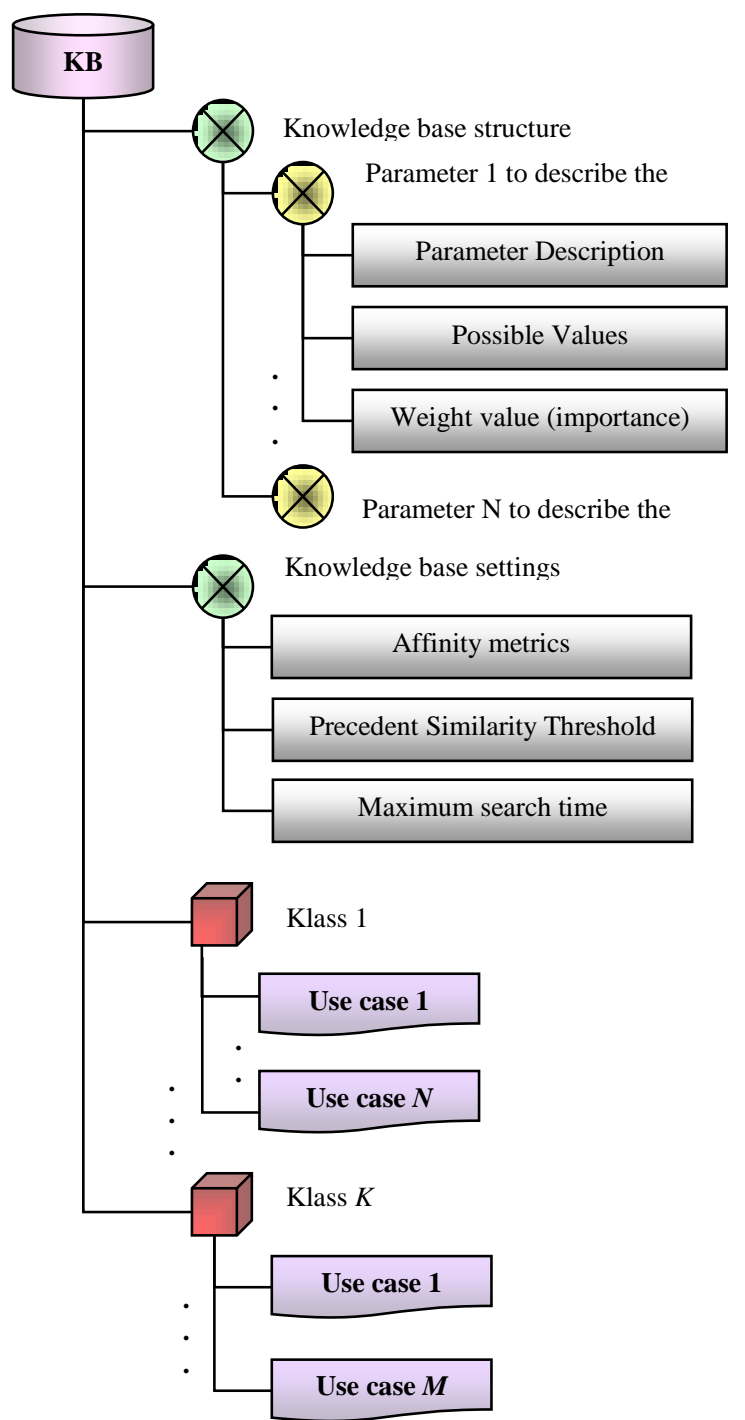

Fig. 5. Knowledge base Structure of Precedent based Neural Network Expert System. 
To increase the speed and efficiency of the search for a precedent in the knowledge base, the full space of precedents is classified by the number of incidents in the precedent.

\section{RESUlts ANALYSIS AND PRACTICAL STRENGHS}

Next main important stage in this research is devoted to an experimental study of the operability and effectiveness of precedent based neural network expert system to solve the problems of subscribers of a cellular communication network. Four groups of indicators were selected as performance indicators: functional, operational, economic indicators and reliability indicators (Table I).

TABLE. I. A LIST OF INDICATORS FOR ASSESSING THE EFFECTIVENESS of PRECEDENT B ASEd NEURAL NETWORK EXPERT SYSTEMS

\begin{tabular}{|l|l|l|}
\hline № & Indicator & Assessment Object \\
\hline 1) Functionality & $\begin{array}{l}\text { The total number of applications } \\
\text { of subscribers }\end{array}$ & System as a whole \\
\hline 2. & Problem Identification Factor & $\begin{array}{l}\text { Use Case Search Subsystem } \\
\text { Neural Network Search } \\
\text { Subsystem } \\
\text { System as a whole }\end{array}$ \\
\hline 3. & $\begin{array}{l}\text { The ratio of unprocessed } \\
\text { applications }\end{array}$ & $\begin{array}{l}\text { Use Case Search Subsystem } \\
\text { Seural Network Search } \\
\text { Subsystem }\end{array}$ \\
\hline System as a whole
\end{tabular}

A technique has been developed for conducting an experiment to check the operability and effectiveness of the developed information system. When testing a precedent based neural network expert system in real conditions, the system was installed on the computers of two operators of the call center of cellular network subscribers. One operator used only the database of customer requests, and another used an additional intelligent subsystem for servicing applications. The experiment was carried out during the week around the clock to take into account the influence of the day of the week and time of day on the frequency of receipt and processing time of subscribers' applications (Table II).

An economic assessment of the effectiveness of the implementation of a neural network expert system based on precedents for solving the problems of subscribers of a cellular network was carried out according to the following particular criteria: development cost, operating cost, annual cost savings, annual economic effect, cost recovery period.

The calculations showed that the costs of creating and maintaining the system pay off for 3.5 years of operation, and the use of intellectual support of the precedent based neural network expert system in the work of the mobile operator gives well enough annual savings.

TABLE. II. Results of Testing PRECEDENT BASEd NeURAl Network EXPERT SYSTEM IN REAL CONDITIONS

\begin{tabular}{|c|c|c|c|c|}
\hline \multirow[b]{2}{*}{ Indicator } & \multicolumn{2}{|c|}{ Intelligent Subsystem } & \multirow{2}{*}{$\begin{array}{l}\text { The non- } \\
\text { intellectual } \\
\text { part of the } \\
\text { system }\end{array}$} & \multirow[b]{2}{*}{$\begin{array}{l}\text { System as } \\
\text { a whole }\end{array}$} \\
\hline & $\begin{array}{l}\text { Use Case } \\
\text { Search } \\
\text { Subsystem }\end{array}$ & $\begin{array}{l}\text { Neural } \\
\text { Network } \\
\text { Search } \\
\text { Subsystem }\end{array}$ & & \\
\hline \multirow{2}{*}{$\begin{array}{l}\text { Total number of } \\
\text { customer } \\
\text { requests }\end{array}$} & 347 & 471 & \multirow{2}{*}{572} & \multirow[t]{2}{*}{1390} \\
\hline & \multicolumn{2}{|l|}{818} & & \\
\hline \multirow{2}{*}{$\begin{array}{l}\text { Problem } \\
\text { Identification } \\
\text { Factor }\end{array}$} & $93,37 \%$ & $100,00 \%$ & \multirow{2}{*}{$91,96 \%$} & \multirow{2}{*}{$95,11 \%$} \\
\hline & \multicolumn{2}{|l|}{$96,69 \%$} & & \\
\hline \multirow{2}{*}{$\begin{array}{l}\text { The ratio of } \\
\text { unprocessed } \\
\text { applications }\end{array}$} & $6,63 \%$ & $0,00 \%$ & \multirow{2}{*}{$8,04 \%$} & \multirow{2}{*}{$4,96 \%$} \\
\hline & \multicolumn{2}{|l|}{$6,63 \%$} & & \\
\hline \multirow{2}{*}{$\begin{array}{l}\text { Problem } \\
\text { Identification } \\
\text { Criteria } \\
\text { Accuracy }\end{array}$} & $96,50 \%$ & $99,72 \%$ & \multirow{2}{*}{$81,33 \%$} & \multirow{2}{*}{$92,52 \%$} \\
\hline & \multicolumn{2}{|l|}{$98,11 \%$} & & \\
\hline \multirow{2}{*}{$\begin{array}{l}\text { Application } \\
\text { processing time } \\
\text { (hours) }\end{array}$} & 35,16 & 24,54 & \multirow{2}{*}{143,00} & \multirow[t]{2}{*}{168} \\
\hline & \multicolumn{2}{|l|}{59,59} & & \\
\hline \multirow{2}{*}{$\begin{array}{l}\text { Duration of } \\
\text { finding a } \\
\text { solution to a } \\
\text { problem }\end{array}$} & 1,39 & 0,59 & \multirow{2}{*}{12,45} & \multirow{2}{*}{4,51} \\
\hline & \multicolumn{2}{|l|}{1,09} & & \\
\hline \multirow{2}{*}{$\begin{array}{l}\text { Average } \\
\text { processing time }\end{array}$} & 5,54 & 3,13 & \multirow{2}{*}{15} & \multirow{2}{*}{7,59} \\
\hline & \multicolumn{2}{|l|}{4,33} & & \\
\hline $\begin{array}{l}\text { The number of } \\
\text { operators }\end{array}$ & \multicolumn{2}{|l|}{1} & 1 & 2 \\
\hline
\end{tabular}




\section{CONCLUSION}

The main scientific results of this research is a theoretical justification, a study of construction methods and the development of a neural network expert system based on precedents for solving the problems of subscribers of a cellular communication network.

It was also investigated modern systems for managing cellular networks and customer relationships. The methods for solving problems that arise during the operation of a cellular communication network are analyzed, and it is concluded that it is advisable to hybridize various intelligent technologies in order to create a single advisory system for solving subscribers' problems. Also a mathematical model of a neural network expert system based on precedents for solving the problems of subscribers of a cellular communication network is developed; the system components and the processes of interaction of its intellectual components are mathematically described. In additional, a mathematical model of a fuzzy knowledge base with a MIMO structure has been built, including knowledge about the problems that subscribers have during the operation of a cellular network. The composition is determined and the characteristic of input and output linguistic variables and their terms is given. Beside the previous mentioned outputs and concludes a mathematical model of a fuzzy neural network output system using a fuzzy controller based on the NNFLC neural network has been developed. The use of a neural network approach to the implementation of fuzzy inference is justified. A module for explaining the solution obtained by the neural network search engine has been implemented.

A neural network expert system based on precedents for solving the problems of subscribers of a cellular network is implemented programmatically. A database of subscriber applications has been developed, which is used both for registering applications and for creating precedents and knowledge base rules on their basis. Also performance indicators of the developed precedent based neural network expert system are determined. An experiment to verify the health and effectiveness of the system. The calculated indicators suggest that the developed precedent based neural network expert system has reliable software, good ability to identify the causes of subscribers' problems, and can identify these causes with a high degree of reliability and high speed.

\section{ACKNOWLEDGEMENT}

This project was funded by the Deanship of Scientific Research (DSR), King Abdulaziz University, Jeddah, under grant No. (DF-749-830-1441). The authors, therefore, gratefully acknowledge DSR technical and financial support.

\section{REFERENCES}

[1] Guimarães, Augusto Junio, et al. "Using fuzzy neural networks to the prediction of improvement in expert systems for treatment of immunotherapy." Ibero-American Conference on Artificial Intelligence. Springer, Cham, 2018.

[2] AlRababah, Ahmad Abdul Qadir. "Watermarking implementation on digital images and electronic signatures." International Journal of Advanced and Applied Sciences 4.10 (2017): 160-164.

[3] Bui, Nhan X., et al. "Reliable data reading with data set screening by error injection." U.S. Patent Application No. 10/262,681.
[4] AlRababah, Ahmad AbdulQadir. "Data Flows Management and Control in Computer Networks." International Journal of Advanced Computer Science and Applications 9.11 (2018): 207-217.

[5] Li, Xiaodong, et al. "Rapid, robust, and reliable blind deconvolution via nonconvex optimization." Applied and computational harmonic analysis 47.3 (2019): 893-934.

[6] Rondinelli, Dennis A. Applied methods of regional analysis: the spatial dimensions of development policy. Routledge, 2019.

[7] Tang, Jinjun, et al. "Lane-changes prediction based on adaptive fuzzy neural network." Expert Systems with Applications 91 (2018): 452-463.

[8] Zhang, Chiya, et al. "Spectrum Sharing of Drone Networks." Handbook of Cognitive Radio (2019): 1279-1304.

[9] AlRABABAH, A.A., Implementation of Software Systems Packages in Visual Internal Structures. Journal of Theoretical \& Applied Information Technology, 2017. 95(19).

[10] Shen, Kai-wen, Xiao-kang Wang, and Jian-qiang Wang. "Multi-criteria decision-making method based on Smallest Enclosing Circle in incompletely reliable information environment." Computers \& Industrial Engineering 130 (2019): 1-13.

[11] Bui, Dac-Khuong, et al. "A modified firefly algorithm-artificial neural network expert system for predicting compressive and tensile strength of high-performance concrete." Construction and Building Materials 180 (2018): 320-333.

[12] AlRababah, Ahmad AbdulQadir. "Lempel-Ziv Implementation for a Compression System Model with Sliding Window Buffer."

[13] Huberman, Gur, Jacob Leshno, and Ciamac C. Moallemi. "An economic analysis of the Bitcoin payment system." Columbia Business School Research Paper 17-92 (2019).

[14] Anand, S. Krishna, TG Sundara Raman, and S. Subramanian. "Implementing a neuro fuzzy expert system for optimising the performance of chemical recovery boiler." International Journal of Artificial Intelligence and Soft Computing 4.2-3 (2014): 249-263.

[15] Mukherjee, Sayandev. "Distribution of downlink SINR in heterogeneous cellular networks." IEEE Journal on Selected Areas in Communications 30.3 (2012): 575-585.

[16] Deliyannis, Theodore, Yichuang Sun, and John Kelvin Fidler. Continuous-time active filter design. CRC press, 2019.

[17] Pelusi, Danilo, et al. "Neural network and fuzzy system for the tuning of Gravitational Search Algorithm parameters." Expert Systems with Applications 102(2018):234-244.

[18] Pearlson, Keri E., and Carol S. Saunders. Managing and using information systems: A strategic approach. John Wiley \& Sons, 2019.

[19] Silva Araújo, Vinícius Jonathan, et al. "Using resistin, glucose, age and bmi and pruning fuzzy neural network for the construction of expert systems in the prediction of breast cancer." Machine Learning and Knowledge Extraction 1.1 (2019): 466-482.

[20] AlRababah, A.A., A. AlShahrani, and B. Al-Kasasbeh, Efficiency Model of Information Systems as an Implementation of Key Performance Indicators. International Journal of Computer Science and Network Security (IJCSNS), 2016. 16(12): p. 139.

[21] Reichling, Markus, and Tim Otto. "The environmental impact of the new economy: Deutsche Telekom, telecommunications services and the sustainable future." The ecology of the new economy. Routledge, 2017. 119-129.

[22] Naderializadeh, Navid, Mohammad Ali Maddah-Ali, and A. Salman Avestimehr. "Cache-aided interference management in wireless cellular networks." IEEE Transactions on Communications 67.5 (2019): 33763387.

[23] Bar-Yam, Yaneer. Dynamics of complex systems. CRC Press, 2019.

[24] Kristjanpoller, Werner, and Marcel C. Minutolo. "A hybrid volatility forecasting framework integrating $\mathrm{GARCH}$, artificial neural network, technical analysis and principal components analysis." Expert Systems with Applications 109 (2018): 1-11.

[25] AlRababah, A.A., A new model of information systems efficiency based on key performance indicator (KPI). management, 2017. 4: p. 8 . 
[26] AL-Qutami, Tareq Aziz, et al. "Virtual multiphase flow metering using diverse neural network ensemble and adaptive simulated annealing." Expert Systems with Applications 93 (2018): 72-85.

[27] Reichling, Markus, and Tim Otto. "The environmental impact of the new economy: Deutsche Telekom, telecommunications services and the sustainable future." The ecology of the new economy. Routledge, 2017. 119-129.

[28] Al-Rababah, Ahmad A., Taghreed AlTamimi, and Najat Shalash. "A New Model for Software Engineering Systems Quality Improvement." Research Journal of Applied Sciences, Engineering and Technology 7.13 (2014): 2724-2728.

[29] Moustafa, Akram, et al. "A New Dynamic Model for Software Testing Quality." Research Journal of Applied Sciences, Engineering and Technology 7.1 (2014): 191-197.

[30] Rendón, Claudio Marco Cartagena, et al. "Proposed Model for Measuring Customer Satisfaction with Telecommunications Services." Mediterranean Journal of Social Sciences 8.2 (2017): 15-25.

[31] Hadi, Mohammed S., et al. "Patient-Centric Cellular Networks Optimization using Big Data Analytics." IEEE Access 7 (2019): 4927949296.

[32] AlRababah, Ahmad. "Digital Image Encryption Implementations Based on AES Algorithm." VAWKUM Transactions on Computer Sciences 13.1 (2017): 1-9.

[33] Smith, Trevor D., et al. "Methods and systems for distributing fiber optic telecommunications services to local area." U.S. Patent No. 8,805,152. 12 Aug. 2014.

[34] Al Ofeishat, H.A. and A.A. Al-Rababah, Real-time programming platforms in the mainstream environments. IJCSNS, 2009. 9(1): p. 197.
[35] Wang, Yueying, Xixiang Yang, and Huaicheng Yan. "Reliable fuzzy tracking control of near-space hypersonic vehicle using aperiodic measurement information." IEEE Transactions on Industrial Electronics (2019).

[36] Chougdali, Sallami, et al. "New air traffic management approach based on expert system and using real-time scheduling algorithms." International Journal of Intelligent Engineering Informatics 4.3-4 (2016): 305-321.

[37] Zahariadis, Nikolaos. "The multiple streams framework: Structure, limitations, prospects." Theories of the Policy Process, Second Edition. Routledge, 2019. 65-92.

[38] AlRababah, Ahmad Abdul Qadir. "On the associative memory utilization in English-Arabic natural language processing." International Journal of Advanced and Applied Sciences 4.8 (2017): 14-18.

[39] Bolz, Ray E. CRC handbook of tables for applied engineering science. CRC press, 2019.

[40] Zurkirch, Manfred, and Inge Reichart. "Environmental Impacts Of Telecommunications Services: Two life-cycle analysis studies." The Ecology of the New Economy. Routledge, 2017. 130-149.

[41] Chen, Min, et al. "Mobility-aware caching and computation offloading in 5G ultra-dense cellular networks." Sensors 16.7 (2016): 974.

[42] Goddard, John B., and Andrew E. Gillespie. "Advanced telecommunications and regional economic development." Managing the city. Routledge, 2017. 84-109.

[43] Al Rababah, Ahmad Abdul Qadir. "Embedded Architecture for Object Tracking using Kalman Filter." JCS 12.5 (2016): 241-245. 